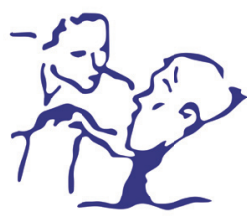

\title{
Definición y recomendaciones para la planificación de decisiones anticipadas: un consenso internacional apoyado por la European Association for Palliative Care (EAPC)
}

\author{
Cristina Lasmarías Martínez ${ }^{* 1-3}$, Sara Delgado Girón ${ }^{4}$, Judit A. C. Rietjens ${ }^{5}$, \\ Ida J. Korfage ${ }^{5}$ y Xavier Gómez-Batiste ${ }^{1,2}$
}

${ }^{1}$ Cátedra de Cures Pal-liatives, Universitat de Vic - Universitat Central de Catalunya (UVIC-UCC), Vic, España. ${ }^{2}$ Institut Català d'Oncologia, Observatorio QUALY, Barcelona, España. ${ }^{3} \mathrm{Grupo}$ de Investigación en Cronicidad de la Cataluña Central (C3RG), Universitat de Vic - Universitat Central de Catalunya (UVIC-UCC), Vic, España. ${ }^{4}$ St. Luke's Hospice, London, United Kingdom. ${ }^{5}$ Department of Public Health, Erasmus University Medical Centre, Rotterdam, Netherlands

Recibido el 22 de diciembre de 2018

Aceptado el 26 de septiembre de 2019

PALABRAS CLAVE

Planificación de

decisiones

anticipadas, Delphi,

consenso

internacional.

\begin{abstract}
Resumen
La Planificación de Decisiones Anticipadas (PDA) - Advance Care Planning (ACP)*- está implementándose exponencialmente en servicios de oncología y otras especialidades a nivel internacional, pero no existe una definición y unas recomendaciones en relación con su uso. Se llevó a cabo un proceso formal Delphi de consenso para contribuir al desarrollo de una definición y proporcionar recomendaciones para su aplicación. El grado de acuerdo entre los 109 expertos (82 de Europa, 16 de Norteamérica y 11 de Australia) que valoraron la definición de PDA y sus 41 recomendaciones osciló entre el 68 y el $100 \%$. La PDA se definió como la capacidad de permitir a las personas atendidas definir objetivos y preferencias sobre tratamientos y atención futuros, discutir esos objetivos y preferencias con los familiares y los profesionales responsables de la atención, y registrar y revisar esas preferencias cuando proceda. Las recomendaciones incluyeron la adaptación de la PDA en base a la predisposición de los individuos, adecuando el contenido de la PDA al deterioro de las condiciones de salud de las personas enfermas, y utilizando figuras de facilitadores/as (no médicos) para el soporte en el proceso de PDA. Presentamos una lista de los resultados obtenidos para facilitar el agrupamiento y la comparación de estos resultados con otros estudios sobre PDA. Creemos que nuestras recomendaciones pueden ser una guía para la práctica clínica, la legislación de PDA y la investigación.
\end{abstract}

La versión original de este artículo fue publicado en inglés en The Lancet Oncology: Rietjens JAC, Sudore RL, Connolly M et al. "Definition and recommendations for advance care planning: an international consensus supported by the European Association for Palliative Care". The Lancet Oncology 18.9 (2017): e543-e551. La versión original se puede visualizar en: https://www.thelancet.com/journals/lanonc/article/PIIS1470-2045(17)30582-X/fulltext

\footnotetext{
\# Para la traducción en este artículo del concepto Advance Care Planning (ACP) se ha utilizado la denominación Planificación de Decisiones Anticipadas (PDA), acuñada en el Modelo Catalán de PDA desde 2015. Se debe tener en cuenta que en España se han utilizado otras denominaciones (PAA, PAAS, PAD), la más reciente es Planificación Compartida de la Atención, consensuada por el Grupo Español de PCA en 2017. El enfoque final, más allá de los matices de las diversas definiciones, es compartido.
}

\section{*Autor para correspondencia:}

Cristina Lasmarías Martínez

Cátedra de Cures Pal-liatives, Universitat de Vic - Universitat Central de Catalunya (UVIC-UCC), Vic, España. Miquel Martí i Pol 1.

Vic 8500. Barcelona, España.

Correo electrónico: clasmarias@iconcologia.net

DOI: 10.20986/medpal.2019.1019/2019

1134-248X/@ 2019 Sociedad Española de Cuidados Paliativos. Publicado por Inspira Network. Todos los derechos reservados. 
KEYWORDS

Advance care

planning, Delphi, international

consensus.

\begin{abstract}
Advance care planning (ACP) is increasingly implemented in oncology and beyond, but a definition of ACP and recommendations concerning its use are lacking. We used a formal Delphi consensus process to help develop a definition of ACP and provide recommendations for its application. Of the 109 experts ( 82 from Europe, 16 from North America, and 11 from Australia) who rated the ACP definitions and its 41 recommendations, agreement for each definition or recommendation was between $68-100 \%$. ACP was defined as the ability to enable individuals to define goals and preferences for future medical treatment and care, to discuss these goals and preferences with family and health-care providers, and to record and review these preferences if appropriate. Recommendations included the adaptation of ACP based on the readiness of the individual; targeting ACP content as the individual's health condition worsens; and, using trained non-physician facilitators to support the ACP process. We present a list of outcome measures to enable the pooling and comparison of results of ACP studies. We believe that our recommendations can provide guidance for clinical practice, ACP policy, and research.
\end{abstract}

Lasmarías Martínez C, Delgado Girón S, Rietjens JAC, Korfage IJ, Gómez-Batiste X. ¿Definición y recomendaciones para la planificación de decisiones anticipadas: un consenso internacional apoyado por la european association for palliative care (EAPC). Revisión crítica. Med Paliat. 2019;26(3):236-249.

\section{Introducción}

La Planificación de Decisiones Anticipadas (PDA) -Advance Care Planning (ACP) en inglés- permite a las personas la realización de planes sobre la atención sanitaria en el futuro. Una sólida evidencia en forma de revisiones sistemáticas muestra que la PDA aumenta la documentación de voluntades anticipadas, promueve la discusión en torno a la futura atención sanitaria y mejora la consistencia entre los cuidados y los objetivos del paciente en varios grupos de pacientes, incluyendo los de enfermedad oncológica ${ }^{1,2}$. La PDA puede mejorar la calidad de la comunicación entre el paciente y los profesionales, disminuir los ingresos hospitalarios no deseados, incrementar el uso de atención paliativa y la satisfacción y la calidad de vida de los pacientes ${ }^{1,2}$.

En 2016, una revisión sistemática ${ }^{3}$ sugirió un amplio apoyo de la PDA entre pacientes con cáncer y sus profesionales referentes. El interés en la PDA continúa creciendo, tal y como indican el incremento en el número de publicaciones científicas, programas, leyes y campañas de concienciación social sobre el tema. Sin embargo, para que la PDA desarrolle todo su potencial, algunos de sus desafíos requieren de un gran consenso.

En primer lugar, el concepto y el contenido de la PDA varían sustancialmente. Originalmente, la PDA fue únicamente conceptualizada como la compleción de las directivas anticipadas, de cara a ser usadas cuando la persona enferma hubiera perdido la competencia para expresarse. Más recientemente, la PDA está siendo considerada como un proceso complejo que incluye reflexiones personales y discusión con los profesionales sobre los deseos de los individuos, la elección de un representante para temas de salud, la compleción de las directivas anticipadas, y los cambios en el sistema sanitario. Este desarrollo ha generado un interés creciente en PDA más allá del estudio en el ámbito geriátrico, como es por ejemplo en oncología ${ }^{3}$.
Además, las iniciativas previas para definir la PDA eran poco generalizables, ya que se restringían principalmente a Norte América o Reino Unido ${ }^{4-7}$ o a grupos específicos de pacientes o de disciplinas ${ }^{6,8}$. En segundo lugar, hay una necesidad de definir las pautas de cuál puede ser el momento adecuado para llevar a cabo una PDA. Por ejemplo, introducir la PDA demasiado pronto puede generar rechazo a involucrarse en el proceso, mientras que involucrar al paciente en la PDA ante una crisis o en un momento cercano a la muerte podría ser demasiado tarde 9 . Un tercer reto en cuanto a la PDA es que el desequilibrio entre preferencias y conocimientos en cuanto a aspectos sanitarios por parte del paciente pueden complicar el manejo de la PDA por parte de los profesionales sanitarios $^{10}$. Finalmente, existe una necesidad urgente de determinar los indicadores de medida más relevantes de cara a la evaluación de la PDA.

En España, a lo largo de los últimos años se han generado iniciativas interesantes en relación con el desarrollo de este concepto, pero con pocos resultados de implementación hasta el momento ${ }^{11,12}$. El caso de la Comunidad Autónoma de Cataluña es tal vez el mejor ejemplo de implementación de la PDA en el sistema asistencial. En 2013 se inicia el desarrollo del modelo catalán de PDA, que incluye un documento conceptual, una guía práctica y un plan formativo ${ }^{13}$; se ha integrado en las políticas de atención a la cronicidad avanzada, incluyendo un espacio específico para el registro de la misma en la historia clínica compartida de los pacientes, así como en la carta de derechos y deberes de los pacientes; en Euskadi el proyecto para impulsar la PDA está basado en la facilitación de las conversaciones en el ámbito de la Atención Primaria y residencias para personas mayores, la formación de los profesionales sanitarios y sociosanitarios, junto con la divulgación comunitaria de los fundamentos y beneficios que ofrece la participación de las personas en la toma de decisiones que afectan a su salud. Por otro lado, en Baleares se está diseñando una propuesta que integre también la PDA en el 
modelo de atención a la cronicidad y en los registros clínicos. Finalmente, desde otras comunidades autónomas han surgido grupos de trabajo y profesionales interesados en el tema que ejercen presión para visibilizar la importancia de este ${ }^{14-17}$, así como legislación diversa que recoja la PDA como un derecho de los usuarios y una obligación de los profesionales. En 2017 surge el Grupo Español de trabajo de la Planificación Compartida de la Atención* que apunta a ser el grupo referente en ámbito nacional. Este grupo consensúa una definición de PDA que integra las publicaciones recientes de consenso terminológico, incluyendo la publicación original de este artículo, y que apuesta por integrar al concepto de PDA el de Toma de decisiones compartidas (Shared-Decision Making). Se define la Planificación compartida de la Atención como un proceso deliberativo, relacional y estructurado, que facilita la reflexión y comprensión de la vivencia de enfermedad y el cuidado entre las personas implicadas, centrado en la persona que afronta una trayectoria de enfermedad, para identificar y expresar sus preferencias y expectativas de atención. Su objetivo es promover la toma de decisiones compartida en relación con el contexto actual y los retos futuros de atención, como aquellos momentos en los que la persona no sea competente para decidir. A pesar de estas iniciativas mencionadas, aún queda mucho camino por recorrer en nuestro país, así como en otros países o contextos de habla hispana.

Hasta la fecha, no hay consenso en relación con la definición de PDA, ni hay ninguna recomendación práctica que sea aplicable a ámbitos culturales y valores personales diversos. Esta falta de acuerdo dificulta el desarrollo de programas de PDA y la evaluación de su efectividad. En este trabajo el objetivo es desarrollar una definición consensuada y presentar recomendaciones para la PDA que puedan ser utilizadas por los profesionales asistenciales, legisladores e investigadores a lo largo de un amplio espectro de poblaciones de pacientes, categorías de enfermedades y culturas.

\section{Métodos}

Un grupo de trabajo internacional compuesto por 15 expertos reconocidos de 8 países (Bélgica, Canadá, Alemania, Irlanda, Italia, Holanda, Reino Unido y EE. UU.) diseñó un estudio Delphi de 5 rondas para construir un consenso sistemático sobre la PDA. La junta de la European Association for Palliative Care (EAPC) impulsó este proyecto de consenso e invitó a Judith A. C. Rietjens (JACR) e Ida J. Korfage (IJK) a dirigirlo, en base a su experiencia en PDA y a sus trabajos comparativos interdisciplinares e internacionales previos.

JACR e IJK invitaron a reconocidos expertos en la PDA con el objetivo de formar un grupo de trabajo internacional y multidisciplinar que incluyera expertos de una amplia variedad de regiones, con experiencia clínica y en investigación en el campo de la oncología, la atención paliativa, geriátrica y en ética. Estos expertos fueron identificados tanto a partir de sus publicaciones y registro de citaciones como a través de contactos de la red profesional de JACR y IJK o de la junta de la EAPC. Las rondas 1 y 5 usaron una metodología cualitativa, mientras que las rondas 2, 3 y 4 usaron evaluación cuantitativa. La Figura 1 muestra el número de participantes y cómo las recomendaciones fueron adaptadas en cada etapa.
Tal y como define el proceso estándar Delphi, las rondas estructuradas se caracterizaron por el anonimato (para evitar que los efectos de conformidad de grupo afectaran los resultados), repetición (permitiendo el cambio de opinión), y feedback controlado (comunicando los resultados de la ronda previa) ${ }^{18,19}$.

\section{Ronda 1}

En junio de 2014, durante una reunión de dos días del Netherlands Institute for Advanced Study (Wassenaar, Netherlands), el grupo de trabajo estableció dos borradores de la definición y cinco dominios principales: elementos/aspectos nucleares, roles y tareas, temporización, legislación y regulación, y evaluación. Se optó por establecer una definición ampliada para que se usara, por ejemplo, en investigación y formación del personal de atención sanitaria, y una definición breve para un uso práctico. Para definir cada dominio en detalle, se crearon subgrupos de trabajo formados por 4 o 5 miembros del grupo principal. Dentro de cada subgrupo, se desarrollaron las recomendaciones basadas, en la medida de lo posible, en la evidencia derivada de la literatura médica y de la opinión de expertos. Este proceso fue llevado a cabo en 2014 y actualizado en 2016. La literatura fue evaluada de tres maneras distintas. En primer lugar, se llevó a cabo una metarrevisión ${ }^{20}$. Esta metarrevisión se basó en la búsqueda en PubMed de publicaciones con el término "Advance Care Planning", incluyendo solo revisiones y metanálisis. La búsqueda se limitó a los campos "título" y "resumen". Esta búsqueda resultó en 89 revisiones y un metanálisis, de los cuales también se revisó el listado de referencias bibliográficas. Estos estudios fueron usados para dar soporte a las recomendaciones iniciales. En segundo lugar, se buscó también en PubMed guías o documentos de posicionamiento publicados con el término "Advance Care Planning" combinado con "guías" o "documentos de posicionamiento".

Se realizó una búsqueda similar en Google, y se chequearon todas las revisiones identificadas (incluyendo la bibliografía), en busca de referencias a guías o documentos de posicionamiento. Esta búsqueda encontró 5 guías de práctica clínica ${ }^{5-8,21}$. En tercer lugar, cada subgrupo de trabajo hizo una revisión específica en PubMed para cada dominio (definición de la PDA, elementos/aspectos nucleares, roles y tareas, temporización, legislación y regulación, y evaluación), combinando el término "Advance care planning" con palabras clave relevantes en cada dominio.

Las definiciones de PDA fueron formuladas en base a 25 definiciones encontradas en la literatura. Adicionalmente, los subgrupos de trabajo pudieron utilizar un estudio previo sobre definición de PDA y clasificación de los resultados que se llevó a cabo principalmente en EE. UU. y Canadá ${ }^{4}$. El borrador de las definiciones de PDA y las recomendaciones fueron discutidas y mejoradas 8 veces por cada subgrupo de trabajo y por el grupo principal (vía email, presencialmente o por teléfono), a lo largo de un año. Este proceso generó en una versión ampliada y una breve de la definición de PDA y 37 recomendaciones preliminares. 
Delphi ronda 1 (junio 2014-agosto 2015). El grupo de trabajo $(n=15)$ redactó dos versiones preliminares de definición de PDA y 37 recomendaciones

Delphi ronda 2 (septiembre 2015-abril 2016). El panel de expertos del Delphi ( $\mathrm{n}=109$ de 144 invitados; respuesta, 76 \%) puntúan las dos definiciones preliminares y las 37 recomendaciones, aportando comentarios. Se determinó acuerdo (mediana) y consenso (IQR). Se hicieron adaptaciones en caso de no haber alto consenso o acuerdo.

10 nuevas recomendaciones fueron añadidas incluyendo 5 nuevos indicadores de resultado

3 recomendaciones fueron eliminadas por redundancia

Dos recomendaciones fueron eliminadas por redundancia y una por baja puntuación

Delphi ronda 3 (mayo 2016-julio 2016). El panel de expertos ( $n=103$ de 109 que completaron la ronda 2; 94 \%) puntuaron el conjunto adaptado de las dos definiciones y las 44 recomendaciones, aportaron comentarios. Se determinó acuerdo y consenso. Se hicieron adaptaciones en caso de no haber alto consenso o acuerdo

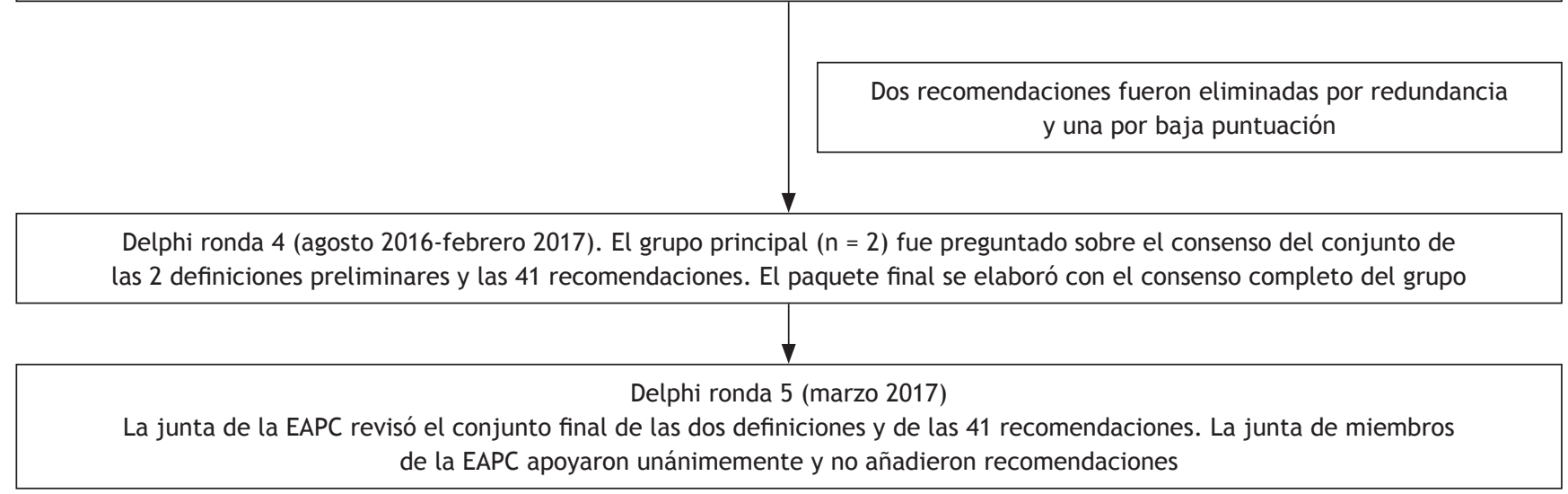

Figura 1 Proceso de consenso Delphi sobre la definición y recomendaciones de PDA.

\section{Ronda 2}

En septiembre de 2015, la definición ampliada y la breve de PDA y las recomendaciones preliminares se presentaron a un panel de expertos a través de un cuestionario online usando el programa LimeSurvey. En un documento separado se envió a los expertos las definiciones y las recomendaciones incluyendo la bibliografía de soporte. El panel de expertos (incluyendo una representación de pacientes) fue identificado a partir de sus publicaciones, del registro de citaciones o a partir de la red de contactos de los miembros del grupo de trabajo o de la junta de la EAPC. En el proceso de selección, se pretendió crear un grupo de expertos en PDA que fuera internacional y multidisciplinar. El panel invitado se compuso de expertos/as en investigación, práctica clínica y legislación en PDA, con formación y experiencia en medicina, enfermería, cuidados paliativos, psicología, ética y legislación. También se incluyó a 9 representantes de pacientes, miembros formados del grupo Expert Voices Group of Marie Curie, y que habían tenido experiencias de primera mano en procesos de final de vida de familiares o amigos. Por ejemplo, un participante fue un estudiante de 19 años que había estado involucrado en el cuidado de tres familiares muy cercanos. Se invitó a 144 expertos/as (de EE.
UU., Canadá, Australia o Europa), de los cuales aceptaron participar 124 (86 \%). De estos/as expertos/as, 109 (76\%) completaron el cuestionario. El apéndice presenta las características del panel del Delphi, procedentes de 14 países. De los 109 expertos/as, 83 trabajaban en la práctica clínica, mayoritariamente en la disciplina médica o enfermera. De los 51 médicos/as, 34 trabajaban en oncología o cuidados paliativos. No se les preguntó qué experiencia profesional tenían trabajando en temas de PDA.

Se preguntó a todos los expertos/as participantes, en relación con la definición y a cada recomendación, que indicaran el grado de acuerdo en una escala Likert de 7 puntos $(1=$ completamente de acuerdo; 2 = de acuerdo; 3 = parcialmente de acuerdo; 4 = indeciso/a; 5 = parcialmente desacuerdo; 6 = desacuerdo; 7 = completamente desacuerdo). Los/as participantes podían además aportar su feedback a las definiciones y a cada recomendación y registrar si consideraban que se habían producido omisiones, escribiendo en un recuadro específico sus propios comentarios. Las respuestas de los/as participantes se usaron para calcular el grado de acuerdo y de consenso ${ }^{22,23}$. El acuerdo fue indicado de dos formas: 1) por el porcentaje de encuestados/as que estaban "de acuerdo" o "completamente de acuerdo" con una definición o recomendación, y 2) por la puntuación 
mediana, que representa en valor percentil 50 de las opiniones. Una mediana inferior indicaba más acuerdo (una mediana de 1 indicaba completamente de acuerdo y una de 2 indicaba acuerdo) ${ }^{24}$. El consenso fue calculado a partir del rango intercuartil (IQR). A menor IQR, mayor consenso: un IQR de 0 o 1 indicaba un consenso muy alto, mientras que un IQR de 2 indicaba alto consenso ${ }^{24}$. Los comentarios en los cuadros de texto fueron analizados en detalle por los subgrupos de trabajo de cada dominio y por JACR y IJK. Las recomendaciones fueron revisadas si se consideró apropiado. Las recomendaciones que recibieron un gran grado de acuerdo o alto consenso fueron aceptadas o sometidas a una revisión de edición menor. El resto de recomendaciones fueron adaptadas respecto a su contenido, redacción u orden, o una combinación de estas, o fueron eliminadas para disminuir redundancia. Las propuestas de adaptación fueron discutidas dentro de los subgrupos de trabajo y del grupo de trabajo principal.

\section{Ronda 3}

De cara a mantener la conformidad entre las rondas, solo aquellos participantes que respondieron al cuestionario online en la ronda 2 fueron invitados a participar en la revisión de las recomendaciones en la ronda 3 . En esta tercera ronda (mayo de 2016), a los 109 participantes de la ronda 2 se les envió el conjunto original de las definiciones y recomendaciones, incluyendo las medianas y las puntuaciones de IQR, y el paquete revisado de definiciones y recomendaciones. De nuevo, los participantes podían expresar el grado de acuerdo con partir de una escala tipo Likert de 1 a 7 y dar su feedback. Si las recomendaciones habían recibido muy alto grado de acuerdo y muy alto nivel de consenso en la segunda ronda, los expertos podían escoger o bien la opción por defecto (es decir, la puntuación mediana de esa recomendación en la ronda anterior) o, alternativamente, puntuar la recomendación de nuevo. De los/as 109 participantes que respondieron en la ronda 2, 103 (94\%) lo hicieron en la ronda 3.

\section{Ronda 4}

Las recomendaciones que recibieron un alto grado de acuerdo (una mediana de 1 ) y muy alto nivel de consenso (un IQR de 0 a 1) fueron aceptadas o sometidas a mínimos ajustes de edición. JACR y IJK adaptaron las otras recomendaciones en base a los comentarios de los participantes. El grupo de recomendaciones revisadas fue enviado a los 15 miembros del grupo principal en agosto de 2016, quienes a su vez indicaron de manera individual si estaban de acuerdo ("sí" o "no") con los cambios sugeridos en cada recomendación adaptada. Si no lo estaban, se proponía a los miembros del grupo que sugiriesen mejoras.

\section{Ronda 5}

El conjunto de recomendaciones y definiciones se adaptó conforme al feedback final del grupo principal. El conjunto completo fue enviado a la junta de dirección de la EAPC.

\section{Resultados}

Las Tablas I y II que se muestran a continuación presentan las definiciones y recomendaciones finales de la PDA. En la ronda 2 , la versión ampliada obtuvo una mediana de 2 (alto grado de acuerdo) y un IQR de 1 (muy alto nivel de consenso), y la versión breve obtuvo una mediana de 2 (alto grado de acuerdo) y un IQR de 2 (alto nivel de consenso). En esta ronda, 28 (76\%) de las 37 recomendaciones obtuvieron muy alto grado de acuerdo y muy alto nivel de consenso (una mediana de 1 y un IQR de 0 o 1).

En la ronda 3, diez recomendaciones fueron añadidas y 3 eliminadas por redundantes (Figura 1). La versión ampliada fue puntuada con una media de 2 (alto acuerdo) y un IQR de 1 (muy alto nivel de consenso), y la definición breve obtuvo una mediana de 2 (alto acuerdo) y un IQR de 1 (muy alto nivel de consenso). En 36 (82\%) de estas recomendaciones, el grado de acuerdo y de consenso fue muy alto.

En la ronda 4, dos recomendaciones fueron eliminadas (una por redundancia y otra por obtener baja puntuación) (Figura 1). De los 15 miembros del grupo principal, 12 miembros puntuaron el grupo restante de 8 recomendaciones que no habían obtenido acuerdo o consenso en la ronda 3. De estas 8 recomendaciones, cuatro obtuvieron acuerdo por todos los miembros, mientras que las otras cuatro obtuvieron acuerdo por parte de 11 de los 12 miembros del grupo. En el feedback se aportaron cambios menores en relación con la redacción. La realización de estos cambios dio como resultado un conjunto de recomendaciones final que obtuvo el consenso de todo el grupo nuclear. El paquete final se compuso de una definición breve de PDA, una definición ampliada y 41 recomendaciones (incluyendo 14 indicadores de resultados de PDA). El conjunto final fue revisado por los miembros de la junta de la EAPC, quienes apoyaron unánimemente el resultado sin ninguna otra sugerencia.

\section{Definición}

El recuadro muestra las definiciones breve y ampliada de PDA. El consenso de la definición breve integra todos los aspectos clave del consenso de la definición ampliada. Un elemento central de las definiciones es que se considera la PDA como un proceso que incluye la identificación de valores y la definición de objetivos y preferencias sobre la atención médica y el cuidado futuros, y que se discuten estos aspectos con el paciente, la familia y los profesionales de la salud. La PDA puede incluir el registro de estas preferencias y la identificación de un representante en la toma de decisiones. Estas preferencias deberían ser revisadas regularmente. Otros puntos clave son que el enfoque de PDA va más allá de aspectos físicos exclusivamente, y que puede incluir preocupaciones sobre aspectos psicológicos, sociales y espirituales. Además, la PDA no debería limitarse a un grupo de pacientes concretos, sino que debería promoverse en personas con capacidad para decidir. Ambas definiciones finales fueron puntuadas con una mediana de 2 (fuerte grado de acuerdo) y un IQR de 1 (muy alto nivel de consenso) en la ronda 3. En total, 91 (88\%) de los participantes (versus 90 [83 \%] en la ronda 2) indicaron que estaban de acuerdo o completamente de acuerdo con la definición ampliada, y 92 (89\%) (versus 71 [65\%] en la ronda 2) con la definición breve. En total, los participantes aporta- 
Tabla I. Definiciones consensuadas de planificación de decisiones anticipadas

\begin{tabular}{cl}
\hline & La planificación de decisiones anticipada (PDA) permite a los individuos con capacidad de \\
& decisión identificar sus valores, reflexionar sobre el significado y consecuencias de escenarios de \\
enfermedad grave, definir objetivos y preferencias para tratamientos y atención médicos \\
futuros, y discutir estos con sus familiares y con los profesionales sanitarios. La PDA aborda las \\
preocupaciones de las personas en los ámbitos físico, psicológico, social y espiritual. Promueve \\
que las personas identifiquen a un representante personal y a registrar y revisar regularmente \\
cualquier preferencia, de manera que estas se tengan en cuenta en caso de que, en algún \\
momento, ellas no pudieran tomar sus propias decisiones
\end{tabular}

Tabla II. Conjunto de recomendaciones finales sobre PDA con los valores otorgados por el panel de expertos en la ronda 3 del Delphi

\begin{tabular}{|c|c|c|c|c|}
\hline & Acuerdo & & $\begin{array}{l}\text { Rango } \\
\text { Intercuartil }{ }^{\ddagger}\end{array}$ & $\begin{array}{l}\text { Comentarios } \\
\text { escritos por el } \\
\text { panel de expertos } \\
\text { en las rondas } 2 \\
\text { y } 3(n) \\
\end{array}$ \\
\hline & Porcentaje $^{*}$ & Mediana $^{\dagger}$ & & \\
\hline \multicolumn{5}{|l|}{ Recomendaciones sobre los aspectos nucleares de la PDA } \\
\hline $\begin{array}{l}\text { 1. El proceso de PDA incluye una exploración de la } \\
\text { comprensión de la persona de la PDA y una explicación de } \\
\text { los objetivos, elementos, beneficios, limitaciones, y } \\
\text { situación legal de la PDA }\end{array}$ & 91 & 1 & 1 & 53 \\
\hline $\begin{array}{l}\text { 2. La PDA debería adaptarse a la disposición de la persona a } \\
\text { participar en el proceso de PDA }{ }^{3,10,25-27}\end{array}$ & 99 & 1 & 0 & 22 \\
\hline $\begin{array}{l}\text { 3. La PDA incluye la exploración de las experiencias } \\
\text { relacionadas con la salud de la persona, conocimiento, } \\
\text { preocupaciones y valores personales en los ámbitos físico, } \\
\text { psicológico, social y espiritual }{ }^{28-30}\end{array}$ & 99 & 1 & 0 & 28 \\
\hline $\begin{array}{l}\text { 4. La PDA incluye explorar los objetivos para la atención } \\
\text { futura }^{28}\end{array}$ & 100 & 1 & 0 & 34 \\
\hline $\begin{array}{l}\text { 5. Cuando sea apropiado, la PDA incluirá información sobre } \\
\text { diagnóstico, curso de la enfermedad, pronóstico, ventajas y } \\
\text { desventajas de posibles tratamientos y opciones de } \\
\text { atención }{ }^{9,31}\end{array}$ & 96 & 1 & 0 & 33 \\
\hline $\begin{array}{l}\text { 6. La PDA podría incluir la clarificación de objetivos y } \\
\text { preferencias para tratamiento médico y atención futuros; si } \\
\text { es necesario, la PDA incluye la exploración de hasta qué } \\
\text { punto tales objetivos y preferencias son realistas }{ }^{28,31,32}\end{array}$ & 83 & 1 & 1 & 55 \\
\hline $\begin{array}{l}\text { 7. La PDA incluye discutir la opción y el rol del representante } \\
\text { personal, quien podría actuar en nombre de la persona } \\
\text { cuando esta no pudiera expresar sus preferencias, así como } \\
\text { por jurisdicción legal local }{ }^{33}\end{array}$ & 94 & 1 & 1 & 50 \\
\hline $\begin{array}{l}\text { 8. La PDA incluye la exploración de hasta qué punto la persona } \\
\text { permite a sus representantes personales considerar su } \\
\text { contexto clínico actual además de las preferencias } \\
\text { previamente manifestadas cuando estos expresen } \\
\text { preferencias en su nombre }{ }^{34-36}\end{array}$ & 74 & 2 & 2 & 31 \\
\hline $\begin{array}{l}\text { 9. La PDA podría incluir la designación de un representante } \\
\text { personal y la documentación que conlleve }{ }^{2,33,37}\end{array}$ & 96 & 1 & 0 & 39 \\
\hline
\end{tabular}


Tabla II. (Cont.) Conjunto de recomendaciones finales sobre PDA con los valores otorgados por el panel de expertos en la ronda 3 del Delphi

\begin{tabular}{|c|c|c|c|c|}
\hline & Acuerdo & & $\begin{array}{l}\text { Rango } \\
\text { Intercuartil }\end{array}$ & $\begin{array}{l}\text { Comentarios } \\
\text { escritos por el } \\
\text { panel de expertos } \\
\text { en las rondas } 2 \\
\text { y } 3(n)\end{array}$ \\
\hline & Porcentaje* & Mediana $^{\dagger}$ & & \\
\hline $\begin{array}{l}\text { 10. La PDA incluye información sobre la opción y rol de un } \\
\text { documento de voluntades anticipadas (DVA) (un } \\
\text { documento para registrar valores, objetivos, y } \\
\text { preferencias a considerar cuando la persona no pueda } \\
\text { expresarlas), así como por jurisdicción legal local }{ }^{33}\end{array}$ & 95 & 1 & 0 & 37 \\
\hline 11. La PDA podría incluir completar un DVA $2,33,38-40$ & 94 & 1 & 0 & 25 \\
\hline $\begin{array}{l}\text { 12. La PDA incluye animar a la persona a facilitar una copia } \\
\text { del DVA a su familia y a los profesionales sanitarios }\end{array}$ & 82 & 1 & 1 & 23 \\
\hline \multicolumn{5}{|l|}{ Recomendaciones sobre roles y tareas } \\
\hline $\begin{array}{l}\text { 13. Los profesionales sanitarios deberían adoptar un abordaje } \\
\text { centrado en la persona al iniciar conversaciones sobre PDA } \\
\text { con personas y, si estas lo desean, con sus familias; este } \\
\text { abordaje requiere adaptar la conversación a los } \\
\text { conocimientos sanitarios de la persona, su estilo de } \\
\text { comunicación y valores personales } \\
22,23,41-45\end{array}$ & 100 & 1 & 0 & 25 \\
\hline $\begin{array}{l}\text { 14. Los profesionales sanitarios deben tener las habilidades } \\
\text { necesarias y mostrar una actitud abierta al hablar sobre } \\
\text { diagnóstico, pronóstico, muerte, y morir con las personas } \\
\text { y sus familias }{ }^{6,28,41,44,46-49}\end{array}$ & 99 & 1 & 0 & 34 \\
\hline $\begin{array}{l}\text { 15. Los profesionales sanitarios deberían ofrecer información } \\
\text { clara y coherente sobre la PDA a las personas y sus familias }{ }^{50}\end{array}$ & 99 & 1 & 0 & 21 \\
\hline $\begin{array}{l}\text { 16. Un facilitador formado y no médico puede dar apoyo a la } \\
\text { persona en el proceso de PDA }\end{array}$ & 91 & 1 & 0 & 46 \\
\hline $\begin{array}{l}\text { 17. El inicio de la PDA (es decir, la exploración de la } \\
\text { experiencia, conocimientos, valores personales y } \\
\text { preocupaciones de la persona enferme) puede producirse } \\
\text { dentro o fuera de las instalaciones sanitarias }{ }^{59,60}\end{array}$ & 98 & 1 & 0 & 31 \\
\hline $\begin{array}{l}\text { 18. Se requieren profesionales sanitarios adecuados para los } \\
\text { elementos clínicos de la PDA, tales como discutir el } \\
\text { diagnóstico, pronóstico, tratamiento, y opciones de } \\
\text { atención, explorando hasta qué punto los objetivos y } \\
\text { preferencias para tratamientos médicos y de atención } \\
\text { futuros son realistas, y documentar la discusión en la } \\
\text { historia clínica médica del paciente }{ }^{61}\end{array}$ & 68 & 2 & 2 & 39 \\
\hline \multicolumn{5}{|l|}{ Recomendaciones sobre la temporización para la PDA } \\
\hline $\begin{array}{l}\text { 19. Las personas pueden iniciar una PDA en cualquier } \\
\text { momento de su vida, pero el contenido puede estar más } \\
\text { orientado al empeoramiento de su estado físico o a su } \\
\text { envejecimiento }\end{array}$ & 96 & 1 & 0 & 39 \\
\hline $\begin{array}{l}\text { 20. Como los valores y preferencias pueden cambiar con el } \\
\text { paso del tiempo, las conversaciones y documentos de la } \\
\text { PDA deberían actualizarse regularmente, a medida que la } \\
\text { condición física de la persona empeore, o su situación } \\
\text { personal cambie, o al ir envejeciendo }{ }^{22,28,62,65-67}\end{array}$ & 99 & 1 & 0 & 18 \\
\hline $\begin{array}{l}\text { 21. Se debería promover la concienciación pública de la PDA, } \\
\text { incluyendo sus objetivos, contenidos, estado legal y cómo } \\
\text { acceder a ella }\end{array}$ & 96 & 1 & 0 & 17 \\
\hline
\end{tabular}


Tabla II. (Cont.) Conjunto de recomendaciones finales sobre PDA con los valores otorgados por el panel de expertos en la ronda 3 del Delphi

\begin{tabular}{|c|c|c|c|c|}
\hline & Acuerdo & & $\begin{array}{l}\text { Rango } \\
\text { Intercuartil }\end{array}$ & $\begin{array}{l}\text { Comentarios } \\
\text { escritos por el } \\
\text { panel de expertos } \\
\text { en las rondas } 2 \\
\text { y } 3(n)\end{array}$ \\
\hline & Porcentaje $^{*}$ & Mediana $^{\dagger}$ & & \\
\hline \multicolumn{5}{|l|}{ Elementos recomendados para la legislación y regulación } \\
\hline $\begin{array}{l}\text { 22. Los DVA requieren un formato estructurado que permita } \\
\text { una fácil identificación de objetivos y preferencias } \\
\text { específicos para situaciones de emergencia, y un espacio } \\
\text { de texto abierto para que las personas puedan describir } \\
\text { sus valores, objetivos y preferencias }{ }^{61,68}\end{array}$ & 80 & 2 & 1 & 57 \\
\hline $\begin{array}{l}\text { 23. Las organizaciones sanitarias deberían desarrollar } \\
\text { activadores potenciales para el inicio de la PDA que } \\
\text { incluyan, aunque no limitados, edad, grado de la } \\
\text { enfermedad, y transiciones en la atención } 9,28,67,69-72\end{array}$ & 95 & 1 & 0 & 31 \\
\hline $\begin{array}{l}\text { 24. Las organizaciones sanitarias necesitan crear sistemas } \\
\text { fiables y seguros de almacenaje de copias de DVA en las } \\
\text { historias clínicas, de modo que puedan recuperarse, } \\
\text { transferir y actualizar de un modo sencillo }\end{array}$ & 97 & 1 & 0 & 29 \\
\hline $\begin{array}{l}\text { 25. Los gobiernos, aseguradoras y organizaciones sanitarias } \\
\text { deberían garantizar financiación y apoyo organizacional } \\
\text { adecuados para la PDA }{ }^{65,76,77}\end{array}$ & 100 & 1 & 0 & 20 \\
\hline $\begin{array}{l}\text { 26. La legislación debería reconocer los resultados del proceso } \\
\text { de PDA (tales como suplir la toma de decisiones o los DVA), } \\
\text { así como asegurar legalmente orientación sobre la toma de } \\
\text { decisiones médicas }\end{array}$ & 91 & 1 & 0 & 37 \\
\hline \multicolumn{5}{|l|}{ Recomendaciones sobre la evaluación } \\
\hline \multicolumn{5}{|l|}{$\begin{array}{l}\text { 27. Dependiendo de los objetivos del estudio o proyecto, se } \\
\text { recomienda valorar los siguientes conceptos: }\end{array}$} \\
\hline $\begin{array}{l}\text { a) Conocimiento de PDA (evaluado por las personas, } \\
\text { familia y profesionales sanitarios) }\end{array}$ & 91 & 1 & 1 & .. \\
\hline $\begin{array}{l}\text { b) Autoeficacia para iniciar una PDA (evaluado por las } \\
\text { personas, familia y profesionales sanitarios) }\end{array}$ & 84 & 2 & 1 & .. \\
\hline $\begin{array}{l}\text { c) Predisposición a iniciar una PDA (evaluado por las } \\
\text { personas, familia y profesionales sanitarios) }\end{array}$ & 92 & 1 & 1 & .. \\
\hline d) Identificación de objetivos y preferencias & 96 & 1 & 0 &. \\
\hline e) Comunicación sobre objetivos y preferencias con la familia & 96 & 1 & 1 & .. \\
\hline $\begin{array}{l}\text { f) Comunicación sobre objetivos y preferencias con } \\
\text { profesionales sanitarios }\end{array}$ & 98 & 1 & 1 & .. \\
\hline g) Identificación del representante personal & 92 & 1 & 1 & .. \\
\hline h) Registro de objetivos y preferencias & 95 & 1 & 0 & .. \\
\hline $\begin{array}{l}\text { i) Revisión de las discusiones y documentos a lo largo del } \\
\text { tiempo }\end{array}$ & 96 & 1 & 0 & .. \\
\hline $\begin{array}{l}\text { j) Hasta qué grado se consideró que la PDA tenía sentido y } \\
\text { resultaba útil (evaluado por las personas, familia, y } \\
\text { profesionales sanitarios) }\end{array}$ & 96 & 1 & 0 & .. \\
\hline $\begin{array}{l}\text { k) Calidad de las conversaciones (evaluado por las } \\
\text { personas, familia, facilitadores y profesionales } \\
\text { sanitarios, o ambos) }\end{array}$ & 90 & 1 & 1 & .. \\
\hline $\begin{array}{l}\text { l) Satisfacción con el proceso de PDA (evaluado por las } \\
\text { personas, familia y profesionales sanitarios) }\end{array}$ & 94 & 1 & 1 & .. \\
\hline m) Sentido de la atención sanitaria & 83 & 2 & 1 &.$\cdot$ \\
\hline $\begin{array}{l}\text { n) Si la atención recibida fue consistente con los objetivos } \\
\text { y preferencias expresados por la persona }\end{array}$ & 92 & 1 & 0 & .. \\
\hline
\end{tabular}


Tabla II. (Cont.) Conjunto de recomendaciones finales sobre PDA con los valores otorgados por el panel de expertos en la ronda 3 del Delphi

\begin{tabular}{|c|c|c|c|c|}
\hline \multirow[t]{2}{*}{ Porcentaje* } & \multicolumn{2}{|l|}{ Acuerdo } & \multirow[t]{2}{*}{$\begin{array}{c}\text { Rango } \\
\text { Intercuartil }\end{array}$} & \multirow[t]{2}{*}{$\begin{array}{l}\text { Comentarios } \\
\text { escritos por el } \\
\text { panel de expertos } \\
\text { en las rondas } 2 \\
\text { y } 3(n)\end{array}$} \\
\hline & Porcentaje $^{*}$ & Mediana $^{\dagger}$ & & \\
\hline $\begin{array}{l}\text { 28. Se recomienda identificar y desarrollar medidas de } \\
\text { resultados basadas en estos conceptos para que puedan } \\
\text { ser agrupados y comparados en estudios o proyectos; tales } \\
\text { medidas deberían tener propiedades psicométricas sólidas, } \\
\text { ser suficientemente breves, y validadas en grupos de } \\
\text { población significativos }\end{array}$ & 89 & 1 & 1 & 37 \\
\hline \multicolumn{5}{|c|}{$\begin{array}{l}\text { PDA: planificación de decisiones anticipadas. DVA: documento de voluntades anticipadas. } \\
\text { "Del total de participantes (109), porcentaje que reportaron el valor de Likert "alto acuerdo" o "acuerdo"; categorías de repuesta: } \\
\text { (1 = completamente de acuerdo; } 2=\text { de acuerdo; } 3=\text { algo de acuerdo; } 4=\text { indeciso/a; } 5=\text { algo desacuerdo; } 6=\text { desacuerdo; } \\
7 \text { = completamente desacuerdo). } \\
\text { †La puntuación en la escala Likert donde los participantes indicaron acuerdo. } \\
\text { fLa puntuación en la escala Likert donde los participantes indicaron consenso. }\end{array}$} \\
\hline
\end{tabular}

ron 97 comentarios con sugerencias para mejorar la definición ampliada, y 88 comentarios sobre la versión breve. Las adaptaciones de ambas definiciones proponían principalmente incluir que las personas debían tener capacidad para involucrarse en el proceso de PDA, la inclusión de aspectos sociales y la importancia de la revisión periódica de las preferencias.

La Tabla II muestra el consenso de las 41 recomendaciones para la PDA, con su acuerdo y puntuaciones medianas respectivas, IQR y el número de comentarios aportados por los participantes. El apéndice ofrece un resumen de las puntuaciones de acuerdo y consenso de las 41 recomendaciones, indicando que la mediana de puntuación y los IQR muestran una desviación hacia valores de muy alto acuerdo y consenso. De las 41 recomendaciones, $36(88 \%)$ recibieron muy alto nivel de consenso y 2 (5\%) recibieron alto grado de acuerdo y alto nivel de consenso. De los 5 dominios de la PDA: 12 recomendaciones se relacionaron con los aspectos nucleares de la PDA, 6 con los roles y tareas, 3 con la temporización, 5 con legislación/ regulación y 15 con la evaluación.

\section{Recomendaciones que obtuvieron muy alto grado de acuerdo y muy alto nivel de consenso}

Las recomendaciones relacionadas con los aspectos de la PDA giraron alrededor de la exploración de la comprensión actual de la persona sobre la PDA y la adecuación del proceso a la disposición de los pacientes para involucrase en el proceso de PDA. Además, se recomienda que la PDA debería incluir la exploración de los valores personales individuales y los objetivos de los cuidados y atención futuros. Cuando sea apropiado, la PDA debería incluir proporcio- nar información médica (por ejemplo, sobre diagnóstico o pronóstico) y clarificar los objetivos y preferencias sobre tratamientos y cuidados futuros, incluyendo la discusión sobre si estos son realistas. Adicionalmente, la conversación sobre la PDA debería incluir la opción de cumplimentar un documento de Voluntades Anticipadas (DVA)**2 y la elección de la persona representante, además de determinar el rol específico de esta persona, en base a la legislación local. LA PDA debería promover que las personas faciliten una copia del DVA a otros miembros de la familia o a los profesionales que les atiendan.

En relación con el dominio de roles y tareas, se recomendó que los profesionales asistenciales adapten la conversación de la PDA al lenguaje de las personas, su estilo de comunicación y sus valores personales. Los profesionales asistenciales deben tener las competencias y estar abiertos a la PDA y a proveer información clara y coherente a la persona atendida y a su familia. Además, se recomienda que un/a facilitador/a o profesional formado/a, que no sea médico, dé soporte a los pacientes en el proceso de PDA y que el inicio de este proceso pueda darse dentro o fuera del ámbito sanitario***3. Para la discusión de aspectos médicos dentro del proceso de PDA (como por ejemplo el diagnóstico, los objetivos de tratamiento y cuidados y la factibilidad de los mismos), es necesaria la participación de profesionales sanitarios.

En relación con la temporalización de la PDA, se recomendó que las personas pueden involucrarse en la PDA en cualquier fase de su vida, pero que el contenido del proceso de la PDA debería estar más dirigido al momento en que la situación de salud de las personas empeora o estas envejecen. En estas circunstancias, las conversaciones en torno a la PDA y los documentos deberían revisarse regularmente

\footnotetext{
"2Se debe tener en cuenta que en España existe terminología diversa para definir las Voluntades Anticipadas: instrucciones previas, testamento vital, directivas anticipadas...

${ }^{* * * 3}$ Esta figura es muy relevante en los modelos anglosajones de PDA, pero no se ha definido tan claramente en nuestro contexto.
} 
porque los valores y preferencias pueden ir cambiando en el tiempo. Es muy recomendable hacer un esfuerzo para aumentar el grado de conocimiento sobre la existencia de la PDA entre la población.

En cuanto a legislación y regulación, se recomendó que las voluntades anticipadas tengan por un lado una estructura (por ejemplo, unas casillas cerradas) y por otro lado campos abiertos de registro. Se anima a los organismos sanitarios a que definan activadores para el inicio de PDA, y que establezcan sistemas seguros y factibles para depositar en las historias clínicas las voluntades anticipadas de los pacientes.

\section{Recomendaciones que recibieron un alto grado de acuerdo y un alto/muy alto nivel de consenso}

Para 5 de las 41 recomendaciones, el grado de acuerdo fue alto (puntuación media 2) y el consenso fue muy alto (IQR 0 o 1) o alto (IQR 2) (Tabla II). Estas 5 recomendaciones incluyeron: la PDA debería incluir la exploración del margen o los límites que el paciente permite a su representante en la toma de decisiones (recomendación 8; alto consenso); la necesidad de tener profesionales sanitarios capacitados para encargarse de los aspectos clínicos de la PDA (recomendación 18; alto consenso); el formato de las voluntades anticipadas (recomendación 22; muy alto nivel de consenso); y la recomendación de evaluar dos constructos-autoeficacia (recomendación27B; muy alto consenso) y el uso de los servicios de cuidados sanitarios (recomendación 27; muy alto nivel de consenso).

Se requieren para los elementos clínicos de la PDA, tales como discutir el diagnóstico, pronóstico, tratamiento y opciones de atención, explorar hasta qué punto los objetivos y preferencias para tratamientos médicos y de atención futuros son realistas, y documentar la discusión en la historia clínica médica del paciente.

\section{Discusión}

Hasta donde sabemos, ha sido elaborado el primer consenso unificado, transcultural e internacional de la definición de PDA y las recomendaciones para su aplicación a partir de un riguroso y amplio estudio Delphi internacional. Las recomendaciones orientan la manera en que la PDA debería realizarse e integrarse en la atención sanitaria y sugiere los indicadores de resultados de la PDA. La mayoría de las recomendaciones recibieron consenso total del panel multidisciplinar de expertos, que también incluía representantes de pacientes. Además, la mayoría generaron consenso en una ronda, mientras que otras lo consiguieron en rondas posteriores. Este nivel de consenso sugiere que las recomendaciones son apropiadas para diversos ámbitos asistenciales, poblaciones de pacientes y culturas. La alta participación en las respuestas de los encuestados implica que el tema tiene alta relevancia en la práctica clínica. También se utilizaron numerosos comentarios de los participantes para mejorar las recomendaciones. Las definiciones y recomendaciones finales suponen una guía importante para llevar a cabo una PDA de alta calidad y se recomienda su uso en estudios próximos y programas clínicos para facilitar la comparación y síntesis de los hallazgos entre los estudios.

Este estudio internacional ofrece mayor generalización que iniciativas previas de definición de PDA o guías o documentos de opinión publicados anteriormente, ya que estos estaban limitados a grupos de pacientes ${ }^{6,8}$ o países o culturas concretos $^{4-7,21}$. Las definiciones y recomendaciones destacan como el foco de PDA se traslada desde la obtención de instrucciones sobre tratamientos a tener en cuenta cuando el individuo pierda la competencia para la toma de decisiones, hacia el diálogo sobre objetivos y preferencias en cuanto a tratamientos futuros a lo largo del espectro de salud-enfer$\operatorname{medad}^{79,80}$. Otro aspecto importante es que el enfoque de la PDA va más allá de aspectos físicos solamente, y puede incluir preocupaciones sobre aspectos psicológicos, sociales y espirituales. Además, la PDA no debería limitarse a un grupo de pacientes, sino que afecta a cualquier grupo de personas con capacidad para decidir. Con este nuevo enfoque, el concepto de PDA ha incrementado su relevancia para muchos grupos de pacientes, como por ejemplo los del área de oncología, enfermedades crónicas y multimorbilidad, y tanto para pacientes como para profesionales asistenciales. Sin embargo, la literatura muestra que en oncología, la PDA continúa limitándose al registro de DVA ${ }^{3}$.

Las definiciones y recomendaciones en este estudio reflejan el valor de la PDA en la provisión de cuidados a personas en diferentes estadios de su enfermedad. La profundidad con la que los profesionales asistenciales, los pacientes y sus allegados están dispuestos o son capaces de conversar sobre aspectos relacionados con la progresión de la enfermedad y el final de la vida, así como el grado en que estas conversaciones están integradas en el sistema asistencial difiere sustancialmente a lo ancho del mundo. Por ello, estas recomendaciones animan a un enfoque individualizado de la PDA (por ejemplo, que se adapta a si el paciente desea participar o no en el proceso) y adaptado a cada estadio de la enfermedad y a las circunstancias legales y culturales de cada sitio. Finalmente, estos hallazgos reflejan la realidad de que, en muchos países, los pacientes pueden expresar sus preferencias de cara a los cuidados, pero existen grados muy diversos de potestad para rechazar tratamientos o limitada capacidad para exigir ciertos tratamientos.

Este estudio presenta varias fortalezas. En primer lugar, la fiabilidad de las recomendaciones se debe al uso de la técnica Delphi. Se siguieron las instrucciones de Conducting and Reporting of Delphi Studies (CREDES) ${ }^{81}$. Este estándar incluía, por ejemplo, la elección de investigadores independientes para la coordinación del estudio, la existencia de criterios de consenso claros, la descripción clara de cómo se llevaría a cabo la síntesis de las respuestas de una ronda para diseñar la siguiente ronda, y la revisión y aprobación del borrador final por parte de una comisión externa antes de su publicación y diseminación. En segundo lugar, siempre que fue posible, se construyeron las definiciones y recomendaciones en base a la evidencia disponible sobre PDA, analizando 90 revisiones publicadas sobre PDA y su respectiva bibliografía. En tercer lugar, la metodología Delphi permitió implicar a una red de 109 expertos de 14 países dispersos geográficamente. Estos participantes representaban varias profesionales y ámbitos asistenciales. En el panel de expertos, también se incluyó a nueve representantes de pacientes. La tasa de respuesta del 76 \% indica que el riesgo de sesgo de selección es bajo. En 
cuarto lugar, aunque los estudios Delphi persiguen determinar el grado de acuerdo que los expertos manifiestan respecto a un constructo (consenso), es escasa una normativa clara sobre niveles de consenso y acuerdos. Se utilizaron puntos de cortes conservadores de grado de acuerdo, añadiendo solidez a los resultados del estudio. En quinto lugar, el elevado nivel de consenso y acuerdo entre el panel de expertos, contribuye a validar nuestros hallazgos. Finalmente, los/as comentarios de los expertos/as fueron analizados sistemáticamente y utilizados para mejorar las definiciones y las recomendaciones resultantes.

Se reconocen las siguientes limitaciones en el estudio. No fue posible llevar a cabo una revisión sistemática de la literatura dada la magnitud de los artículos publicados sobre el tema de la PDA, con conceptos, preguntas de investigación y metodologías diversas. Seguramente estas recomendaciones tendrán que ser actualizadas a medida que se disponga de más evidencia. Además, la evidencia científica y la visión de los expertos provienen principalmente de países o regiones desarrolladas, como Europa, Norteamérica y Australia. No hay representación de Asia, Sur América o África. Es probable que se requieran adaptaciones culturales de las definiciones y recomendaciones si se desea aplicarlas a regiones que no estaban representadas en el panel de expertos del Delphi. En estos casos, se recomienda llevar a cabo un estudio Delphi adicional para determinar recomendaciones que representen adecuadamente a estas regiones. Finalmente, las definiciones y recomendaciones necesitan ser validadas en diferentes poblaciones. Si el uso de las recomendaciones mejorará los procesos y los resultados en el cuidado de las personas es un tema que requiere futuros estudios.

Como pasos futuros, se recomienda la traducción, diseminación e implementación de estas definiciones y recomendaciones para su uso en la práctica y en el desarrollo de políticas y legislación relacionadas con el tema. También es aconsejable la evaluación del uso de estas recomendaciones en la práctica clínica y su legislación. Trabajos futuros podrían incluir la prioridad de la práctica en los ámbitos sugeridos en las recomendaciones ${ }^{82}$. Se está trabajando para definir resultados sobre dimensiones y constructos de PDA, mediante un estudio Delphi aparte, para desarrollar un conjunto de recomendaciones que estandaricen los constructos e instrumentos para la PDA ${ }^{83,84}$. Además, se anima a la identificación de instrumentos de evaluación para la medida de resultados de la PDA. Adicionalmente, para reforzar una amplia aplicación de estas recomendaciones, se pretende proporcionar recomendaciones generales para todas las disciplinas. Futuros trabajos podrían centrarse en elaborar recomendaciones específicas según disciplinas, sistemas asistenciales y jurisdicciones locales. Se recomienda poner especial atención en la PDA en el contexto de capacidad limitada, ya que este aspecto estuvo fuera del objetivo del presente estudio.

\section{Conclusión}

Este amplio estudio Delphi internacional llegó a un consenso sobre la definición de la PDA y las recomendaciones para su aplicación práctica. Esta revisión representa un primer paso importante para dar claridad a próximas normativas, legisla- ción o investigaciones en el campo de la PDA. Esperamos que estas recomendaciones generen un efecto catalizador para el beneficio futuro de los pacientes y sus familiares, facilitando una atención a pacientes con cáncer, y otras patologías, que esté alineado con sus preferencias y objetivos, y que contribuya a una mejora en su calidad de vida.

\section{Nota}

Los autores originales del artículo son:

J.A.C. Rietjens, $\mathrm{PhD}^{1}$, Prof. R.L. Sudore, $\mathrm{MD}^{2-3}$, M. Connolly, PhD ${ }^{4}$, Prof. J.J. van Delden, MD5 , Prof. M.A. Drickamer, $M D^{6-7}, M$. Droger, $M \mathrm{MC}^{1}$, Prof. A. van der Heide, $M D^{1}$, Prof. D.K. Heyland, $M D^{8}$, D. Houttekier, $\mathrm{PhD}^{9}$, D.J.A. Janssen, $\mathrm{MD}^{10-11}$, L. Orsi, MD ${ }^{12}$, Prof. S. Payne, $\mathrm{PhD}^{13}$, Prof. J. Seymour, $\mathrm{PhD}^{14}$, R.J. Jox ${ }^{15-16}$, I.J. Korfage, $\mathrm{PhD}^{1}$ on behalf of the European Association for Palliative Care (EAPC) Lancet Oncol 2017; 18: e543-51 Vol 18 September 2017.

'Department of Public Health, Erasmus MC Rotterdam, Rotterdam, the Netherlands ${ }^{2}$ Division of Geriatrics, Department of Medicine, University of California, San Francisco, California, US; ${ }^{3}$ San Francisco Veterans Affairs Medical Center, San Francisco, California, US ${ }^{4}$ UCD School of Nursing, Midwifery and Health Systems, University College Dublin, Dublin, Ireland 5 Julius Center, Department of Medical Humanities, University Medical Center Utrecht, Utrecht, the Netherlands; ${ }^{6}$ School of Medicine, University of North Carolina, Chapel Hill (NC), US; ${ }^{7}$ School of Medicine, Yale University, New Haven (CT), US ${ }^{8}$ Department of Critical Care Medicine, Queen's University, Kingston, ON, Canada. ${ }^{9}$ End-of-Life Care Research Group, Vrije Universiteit Brussel (VUB) \& Ghent University, Brussels, Belgium ${ }^{10}$ Department of Research \& Education, CIRO, Centre of expertise for chronic organ failure, Horn, The Netherlands; "Centre of Expertise for Palliative Care, Maastricht University Medical Centre (MUMC+), Maastricht, The Netherlands; ${ }^{12}$ Palliative Care Unit, C. Poma Hospital, via Lago Paiolo 10, Mantova, Italy; ${ }^{13}$ International Observatory on End of Life Care, Division of Health Research, Lancaster University, Lancaster, UK; ${ }^{14} \mathrm{~S}$ Chool of Nursing and Midwifery, University of Sheffield, Sheffield, UK ${ }^{15}$ Institute of Ethics, History and Theory of Medicine, Ludwig-Maximilians University of Munich, Munich, Germany; ${ }^{16}$ Geriatric Palliative Care, Centre Hospitalier Universitaire Vaudois, University of Lausanne, Lausanne, Switzerland.

\section{Traducción}

La traducción de este artículo al castellano ha sido realizada por: Cristina Lasmarías, Sara Ela, Sara Delgado y Brad London.

\section{Conflicto de intereses}

Las autoras declaran no tener ningún conflicto de interés en la publicación de este artículo. Asimismo, no se ha recibido financiación alguna para la realización de esta traducción. 


\section{Agradecimientos}

Agradecemos a las personas componentes del GET_PCA por su contribución a este trabajo: Virginia Elena Carrero, Sara Delgado, Julia Fernández, Helena García, Nani Granero, Francisco Javier Júdez, Cristina Lasmarías, Nuria Pérez de Lucas, Iñaki Saralegui y Tayra Velasco.

\section{Bibliografía}

1. Brinkman-Stoppelenburg A, Rietjens JA, van der Heide A. The effects of advance care planning on end-of-life care: systematic review. Palliat Med. 2014;28:1000-25.

2. Houben CH, Spruit MA, Groenen MT, Wouters EF, Janssen DJ. Efficacy of advance care planning: a systematic review and meta-analysis. J Am Med Dir Assoc. 2014;15:477-89.

3. Johnson S, Butow P, Kerridge I, Tattersall M. Advance care planning for cancer patients: a systematic review of perceptions and experiences of patients, families, and healthcare providers. Psychooncology. 2016;25:362-86.

4. Sudore RL, Lum HD, You JJ, Hanson LC, Meier DE, Pantilat SZ, et al. Defining advance care planning for adults: a consensus definition from a multidisciplinary Delphi panel. J Pain Symptom Manage. 2017;53:821-32.

5. Institute of Medicine. Dying in America: improving quality and honoring individual preferences near the end of life. Washington, DC: The National Academies Press; 2015.

6. Conroy S, Fade P, Fraser A, Schiff R; Guideline Development Group. Advance care planning: concise evidence-based guidelines. Clin Med. 2009;9:76-9.

7. National End of Life Care Programme. Capacity, care planning and advance care planning in life limiting illness; June 2011. Available in: http://www.ncpc.org.uk/sites/default/files/ ACP_Booklet_June_2011.pdf (accessed April 7, 2017).

8. Schrijvers D, Cherny NI, Group EGW. ESMO Clinical Practice Guidelines on palliative care: advanced care planning. Ann Oncol. 2014;25(suppl 3):iii138-42.

9. Billings JA, Bernacki R. Strategic targeting of advance care planning interventions: the Goldilocks phenomenon. JAMA Intern Med. 2014;174:620-4.

10. Barclay JS, Blackhall LJ, Tulsky JA. Communication strategies and cultural issues in the delivery of bad news. J Palliat Med. 2007;10:958-77.

11. Barrio IM, Simón P, Pascau MJ. El papel de la enfermera en la planificación anticipada de las decisiones: más allá de las instrucciones previas o voluntades anticipadas. Enferm Clin. 2004;14:235-41.

12. Altisent $R$, Judez J. El reto de la planificación anticipada de la atención al final de la vida en España. Med Paliat. 2016; 23:163-4.

13. Lasmarías C, Aradilla-Herrero A, Santaeugènia S, Blay C, Delgado $S$, Ela $S$, et al. Development and implementation of an advance care planning program in Catalonia, Spain. Palliat Support Care. 2019;17:415-24.

14. Carrero Planes V, Navarro Sanz R, Serrano Font M. Planificación adelantada de los cuidados en pacientes con enfermedades crónicas y necesidad de atención paliativa. Med Paliat. 2016;23:32-41.

15. Granero-Moya N, Frías-Osuna A, Barrio-Cantalejo IM, RamosMorcillo AJ. Dificultades de las enfermeras de atención primaria en los procesos de planificación anticipada de las decisiones: un estudio cualitativo. Aten Primaria. 2016;48:649-56.

16. Simón P, Tamayo MI, Esteban MS, Blanco N, Fernández A, Gómez $M$, et al. Planificación anticipada de las decisiones: Guía de apoyo para los profesionales. Sevilla: Junta de Andalucía, Consejería de Salud y bienestar Social; 2013.
17. Júdez J. La facilitación como herramienta básica de planificación anticipada de la asitencia sanitaria. Fundamentos del programa "KAYRÓS-conversaciones que ayudan" (Inspirado en Respecting Choices ${ }^{\oplus}$. Tesis Doctoral. Murcia: Universidad de Murcia; 2014. Disponible en: http://digitum.um.es/xmlui/handle/10201/40846.

18. De Vet E, Brug J, De Nooijer J, Dijkstra A, De Vries NK. Determinants of forward stage transitions: a Delphi study. Health Educ Res. 2005;20:195-205.

19. Biondo PD, Nekolaichuk CL, Stiles C, Fainsinger R, Hagen NA. Applying the Delphi process to palliative care tool development: lessons learned. Support Care Cancer. 2008;16(8):935-42.

20. Francke AL, Smit MC, de Veer AJE, Mistiaen P. Factors influencing the implementation of clinical guidelines for health care professionals: systematic meta-review. BMC Med Inform Decis Mak. 2008;8:38.

21. Australian Medical Association. Position statement on end of life care and advance care planning 2014. Available in: https: // ama.com.au/system/ tdf/documents/AMA_position_statement_on_end_of_life_care_and_advance_care_planning_2014. pdf?file=1\&type=node\&id=40573 (accessed April 7, 2017).

22. Jones J, Hunter D. Consensus methods for medical and health services research. BMJ. 1995;311:376-80.

23. Linstone HA, Turoff $M$. The Delphi method: techniques and applications. Boston: Addison-Wesley; 1975.

24. van der Steen JT, Radbruch L, Hertogh CM, van der Steen JT, Radbruch L, Hertogh CM, de Boer ME, Hughes JC, Larkin P, et al; European Association for Palliative Care. White paper defining optimal palliative care in older people with dementia: a Delphi study and recommendations from the European Association for Palliative Care. Palliat Med. 2014;28:197-209.

25. Parker SM, Clayton JM, Hancock K, Walder S, Butow PN, Carrick $S$, et al. A systematic review of prognostic/end-of-life communication with adults in the advanced stages of a life-limiting illness: patient/caregiver preferences- for the content, style, and timing of information. J Pain Symptom Manage. 2007;34:81-93.

26. Fried TR, Bullock K, lannone L, O'Leary JR. Understanding advance care planning as a process of health behavior change. $J$ Am Geriatr Soc. 2009;57:1547-55.

27. Fried TR, Redding CA, Robbins ML, Paiva A, O'Leary JR, lannone L. Stages of change for the component behaviors of advance care planning. J Am Geriatr Soc. 2010;58:2329-36.

28. Bernacki RE, Block SD; American College of Physicians High Value Care Task Force. Communication about serious illness care goals: a review and synthesis of best practices. JAMA Intern Med. 2014;174:1994-2003.

29. Steinhauser KE, Christakis NA, Clipp EC, McNeilly M, McIntyre L, Tulsky JA. Factors considered important at the end of life by patients, family, physicians, and other care providers. JAMA. 2000;284:2476-82.

30. Weiner JS, Cole SA. Three principles to improve clinician communication for advance care planning: overcoming emotional, cognitive, and skill barriers. J Palliat Med. 2004;7:817-29.

31. Mack JW, Weeks JC, Wright AA, Block SD, Prigerson HG. End-oflife discussions, goal attainment, and distress at the end of life: predictors and outcomes of receipt of care consistent with preferences. J Clin Oncol. 2010;28:1203-8.

32. Messinger-Rapport BJ, Baum EE, Smith ML. Advance care planning: beyond the living will. Cleve Clin J Med. 2009;76:276-85.

33. Silveira MJ, Kim SY, Langa KM. Advance directives and outcomes of surrogate decision making before death. N Engl J Med. 2010;362:1211-18.

34. Sehgal A, Galbraith A, Chesney M, Schoenfeld P, Charles G, Lo B. How strictly do dialysis patients want their advance directives followed? JAMA. 1992;267:59-63.

35. Sulmasy DP, Hughes MT, Thompson RE, Astrow AB, Terry PB, Kub $J$, et al. How would terminally ill patients have others make decisions for them in the event of decisional incapacity? A longitudinal study. J Am Geriatr Soc. 2007;55:1981-8.

36. Wendler D, Rid A. Systematic review: the effect on surrogates of making treatment decisions for others. Ann Intern Med. 2011;154:336-46. 
37. Sudore RL, Fried TR. Redefining the "planning" in advance care planning: preparing for end-of-life decision making. Ann Intern Med. 2010;153:256-61.

38. Glaudemans JJ, Moll van Charante EP, Willems DL. Advance care planning in primary care, only for severely ill patients? A structured review. Fam Pract. 2015;32:16-26.

39. Teno JM, Gruneir A, Schwartz Z, Nanda A, Wetle T. Association between advance directives and quality of end-of-life care: a national study. J Am Geriatr Soc. 2007;55:189-94.

40. Hickman SE, Nelson CA, Moss AH, Tolle SW, Perrin NA, Hammes BJ. The consistency between treatments provided to nursing facility residents and orders on the physician orders for lifesustaining treatment form. J Am Geriatr Soc. 2011;59:2091-99.

41. Clayton JM, Hancock K, Parker S, Butow PN, Walder S, Carrick $\mathrm{S}$, et al. Sustaining hope when communicating with terminally ill patients and their families: a systematic review. Psychooncology. 2008;17:641-59.

42. Sanders JJ, Robinson MT, Block SD. Factors impacting advance care planning among african americans: results of a systematic integrated review. J Palliat Med. 2016;19:202-27.

43. Baker ME. Economic, political and ethnic influences on end-oflife decision-making: A decade in review. J Health Soc Policy. 2002;14:27-39.

44. Patel K, Janssen DJ, Curtis JR. Advance care planning in COPD. Respirology. 2012;17:72-8.

45. Bullock K. The influence of culture on end-of-life decision making. J Soc Work End Life Palliat Care. 2011;7:83-98.

46. Szmuilowicz E, El-Jawahri A, Chiappetta L, Kamdar M, Block S. Improving residents' end-of-life communication skills with a short retreat: a randomized controlled trial. J Palliat Med. 2010;13:439-52.

47. Fallowfield L, Jenkins V, Farewell V, Saul J, Duffy A, Eves R. Efficacy of a Cancer Research UK communication skills training model for oncologists: a randomised controlled trial. Lancet. 2002;359:650-6.

48. Cavalieri TA, Latif W, Ciesielski J, Ciervo CA Jr, Forman LJ. How physicians approach advance care planning in patients with mild to moderate Alzheimer's disease. J Am Osteopath Assoc. 2002;102:541-44.

49. Back AL, Arnold RM, Baile WF, Fryer-Edwards KA, Alexander SC, Barley GE, et al. Efficacy of communication skills training for giving bad news and discussing transitions to palliative care. Arch Intern Med. 2007; 167:453-60.

50. Stewart F, Goddard C, Schiff R, Hall S. Advanced care planning in care homes for older people: a qualitative study of the views of care staff and families. Age Ageing. 2011;40:330-5.

51. Hickman SE, Keevern E, Hammes BJ. Use of the physician orders for life-sustaining treatment program in the clinical setting: a systematic review of the literature. J Am Geriatr Soc. 2015;63:341-50.

52. Detering KM, Hancock AD, Reade MC, Silvester W. The impact of advance care planning on end of life care in elderly patients: randomised controlled trial. BMJ. 2010;340:c1345.

53. Molloy DW, Guyatt GH, Russo R, Goeree R, O’Brien BJ, Bédard $M$,et al. Systematic implementation of an advance directive program in nursing homes: a randomized controlled trial. JAMA. 2000;283:1437-44.

54. Morrison RS, Chichin E, Carter J, Burack O, Lantz M, Meier DE. The effect of a social work intervention to enhance advance care planning documentation in the nursing home. J Am Geriatr Soc. 2005;53:290-4.

55. Chan HYL, Pang S. Let me talk-an advance care planning programme for frail nursing home residents. J Clin Nurs. 2010;19:3073-84.

56. Kirchhoff KT, Hammes BJ, Kehl KA, Briggs LA, Brown RL. Effect of a disease-specific advance care planning intervention on endof-life care. J Am Geriatr Soc. 2012;60:946-50.
57. Song MK, Kirchhoff KT, Douglas J, Ward S, Hammes B. A randomized, controlled trial to improve advance care planning among patients undergoing cardiac surgery. Med Care. 2005;43:104953.

58. Litzelman DK, Inui TS, Griffin WJ, Perkins A, Cottingham $\mathrm{AH}$, Schmitt-Wendholt KM, et al. Impact of community health workers on elderly patients' advance care planning and health care utilization: moving the dial. Med Care. 2017;55:319-26.

59. Austin CA, Mohottige D, Sudore RL, Smith AK, Hanson LC. Tools to promote shared decision making in serious illness: systematic review. JAMA Intern Med. 2015;175:1213-21.

60. Jain A, Corriveau S, Quinn K, Gardhouse A, Vegas DB, You JJ. Video decision aids to assist with advance care planning: systematic review and meta-analysis. BMJ Open. 2015;5e:007491.

61. Robinson L, Dickinson C, Bamford C, Clark A, Hughes J, Exley C. A qualitative study: professionals' experiences of advance care planning in dementia and palliative care, 'a good idea in theory but...'. Palliat Med. 2013;27:401-8.

62. Auriemma CL, Nguyen CA, Bronheim R, Kent S, Nadiger S, Pardo $D$, et al. Stability of end-of-life preferences: a systematic review of the evidence. JAMA Intern Med. 2014;174:1085-92.

63. van der Steen JT, van Soest-Poortvliet MC, Hallie-Heierman M, Onwuteaka-Philipsen BD, Deliens L, de Boer ME, et al. Factors associated with initiation of advance care planning in dementia: a systematic review. J Alzheimers Dis. 2014;40:743-57.

64. Malcomson H, Bisbee S. Perspectives of healthy elders on advance care planning. J Am Acad Nurse Pract. 2009;21:18-23.

65. Janssen DJA, Spruit MA, Schols JMGA, Cox B, Nawrot TS, Curtis $J R$, et al. Predicting changes in preferences for life-sustaining treatment among patients with advanced chronic organ failure. Chest. 2012;141:1251-9.

66. Janssen DJA, Spruit MA, Schols JMGA, van der Sande FM, Frenken LA, Wouters EFM. Insight into advance care planning for patients on dialysis. J Pain Symptom Manage. 2013;45:104-13.

67. Mullick A, Martin J, Sallnow L. An introduction to advance care planning in practice. BMJ. 2013;347:f6064.

68. Flo E, Husebo BS, Bruusgaard P, Gjerberg E, Thoresen L, Lillemoen $L$, et al. A review of the implementation and research strategies of advance care planning in nursing homes. BMC Geriatr. 2016;16:24.

69. Kirkpatrick JN, Hauptman PJ, Goodlin SJ. Bundling informed consent and advance care planning in chronic cardiovascular disease: we need to talk. JAMA Intern Med. 2015;175:5-6.

70. Dow LA, Matsuyama RK, Ramakrishnan V, Kuhn L, Lamont $E B$, Lyckholm L, et al. Paradoxes in advance care planning: the complex relationship of oncology patients, their physicians, and advance medical directives. J Clin Oncol. 2010;28:299-304.

71. Lamont EB, Siegler M. Paradoxes in cancer patients' advance care planning. J Palliat Med. 2000;3:27-35.

72. Gott M, Gardiner C, Small N, Payne S, Seamark D, Barnes S, et al. Barriers to advance care planning in chronic obstructive pulmonary disease. Palliat Med. 2009;23:642-8.

73. Wilson DM, Cohen J, Deliens L, Hewitt JA, Houttekier D. The preferred place of last days: results of a representative population-based public survey. J Palliat Med. 2013;16:502-8.

74. Yung VY, Walling AM, Min L, Wenger NS, Ganz DA. Documentation of advance care planning for community-dwelling elders. J Palliat Med. 2010;13:861-7.

75. Skinner I, Smith C, Jaffray L. Realist review to inform development of the electronic advance care plan for the personally controlled electronic health record in Australia. Telemed J E Health. 2014;20:1042-8.

76. Johnson M, Attree M, Jones I, Al Gamal E, Garbutt D. Diagnosis, prognosis and awareness of dying in nursing homes: towards the Gold Standard? Int J Older People Nurs. 2014;9:95-105.

77. Ke LS, Huang X, O'Connor M, Lee S. Nurses' views regarding implementing advance care planning for older people: a sys- 
tematic review and synthesis of qualitative studies. J Clin Nurs. 2015;24:2057-73.

78. Bausewein C, Daveson BA, Currow DC, Downing J, Deliens $\mathrm{L}$, Radbruch L, et al. EAPC White Paper on outcome measurement in palliative care: improving practice, attaining outcomes and delivering quality services-recommendations from the European Association for Palliative Care (EAPC) Task Force on Outcome Measurement. Palliat Med 2015;30:6-22.

79. Sabatino CP. The evolution of health care advance planning law and policy. Milbank Q. 2010;88:211-39.

80. Seymour J. Advance care planning for the end of life: an overview. In: Thomas K, Lobo B, eds. Advance care planning in end of life care. $1 .^{a}$ ed. Oxford: Oxford University Pres; 2011. p. 16-27.
81. Jünger S, Payne SA, Brine J, Radbruch L, Brearley SG. Guidance on Conducting and Reporting Delphi Studies (CREDES) in palliative care: recommendations based on a methodological systematic review. Palliat Med. 2017;31:684-706.

82. Johnson AP, Hanvey L, Baxter S, Daren K; Canadian Researchers at the End of Life Network. Development of advance care planning research priorities: a call to action. J Palliat Care. 2013;29:99-106.

83. Howard M, Bonham AJ, Heyland DK, Sudore R, Fassbender K, Robinson $\mathrm{CA}$, et al. Measuring engagement in advance care planning: a crosssectional multicentre feasibility study. BMJ Open. 2016;6:e010375.

84. Sudore RL, Heyland DK, Barnes DE, Howard M, Fassbender $\mathrm{K}$, Robinson CA, et al. Measuring advance care planning: optimizing the advance care planning engagement survey. J Pain Symptom Manage. 2017;53:669-81. 